\title{
Estudio longitudinal: Cáncer de Ovario en el Instituto Nacional de Oncología y Radiobiología de Cuba (INOR), resultados del tratamiento.
}

*Correspondencia: igarcia@hchr.vcl.sld.cu Teléfono [53] 042210023

Conflicto de intereses: Los autores declaran no tener conflictos de intereses.

Fondos: Ver la página 160

Recibido: 1 Abril 2016

Aceptado: 29 Marzo 2018

Publicado: 30 Agosto 2018

Membrete bibliográfico:

García I, Linchenat A, Pérez R, Silveira M, Esperón R, Aguilar K. Estudio longitudinal: Cáncer de Ovario en el Instituto Nacional de Oncología y Radiobiología de Cuba (INOR), resultados del tratamiento. Rev. Oncol. Ecu 2018;28(2):150162.

DOI: https://doi.org/10.33821/106

Copyright García, et al. Este artículo es distribuido bajo los términos de Creative Commons Attribution License, el cual permite el uso y redistribución citando la fuente y al autor original.

\section{Longitudinal study: Ovarian cancer at the National Institute of Oncology and Radiobiology of Cuba (INOR), treatment results.}

Iván García Alonso1* iD, Alejandro Linchenat Lambert², Roberto Pérez Martínez³, Mario Silveira Pablos², Roberto Esperón Noa², Kenia Aguilar Vela de Oro².

1. Hospital Universitario "Celestino Hernández Robau" Santa Clara-Cuba.

2. Instituto Nacional de Oncología y Radiobiología (INOR). La Habana-Cuba.

3. Hospital "Gustavo Aldereguia Lima". Cienfuegos-Cuba.

\section{Resumen}

Introducción: El cáncer de ovario es una causa común de muerte entre las mujeres que desarrollan neoplasias ginecológicas. La supervivencia depende de factores clínicos y del tipo de cirugía. El objetivo del presente estudio es describir la supervivencia de un grupo de pacientes con diagnóstico de cáncer de ovario tomando en cuenta varios factores.

Métodos: El presente estudio longitudinal retrospectivo se realizó en mujeres con cáncer de ovario tratadas en el Instituto Nacional de Oncología y Radiobiología, La Habana-Cuba entre el 1 de enero y el 31 de diciembre de 2005, con seguimiento de por lo menos 5 años. Las variables fueron edad, tipo histológico del tumor, etapa clínica de la FIGO, grado de diferenciación celular, valores del biomarcador CA-125, tipo de cirugía realizada, respuesta a la quimioterapia utilizada, supervivencia, recurrencias. El paquete estadístico utilizado fue SPSS 11.0 para Windows. Se aplicaron pruebas estadísticas como la prueba no paramétrica de Kruskal-Wallis, la prueba no paramétrica de MannWhitney y la prueba de Chi-cuadrado de Pearson.

Resultados: Se registraron 29 casos de mujeres con cáncer de ovario. Once casos (37.9\%) fueron en menores de 45 años. En Etapa clínica III, 18 casos (62.1\%). De grado indiferenciado en 17 casos (58.6 $\%) .16$ casos (55.2\%) fueron del tipo histológico seroso. El valor del CA- 125 fue $>35 \mathrm{U} / \mathrm{mL}$ en 13 casos (61.9\%). Las mujeres con etapa clínica I, tuvieron un Intervalo Libre de la Enfermedad (ILE) de 46 meses versus 27 meses en mujeres con etapa III y 12 meses en etapa IV $(P>0.05)$. El grado de 
diferenciación celular Bien diferenciado determinó estadísticamente el mejor ILE: 55 meses versus 27 meses en el grado moderadamente diferenciado y de 21 meses en el grado indiferenciado $(P=0.025)$. El tipo histológico así como los niveles de CA-125 no determinaron diferencias estadísticas de ILE, así como en Supervivencia Global Media (SGM). Según el tipo de tratamiento no existieron diferencias estadísticas significativas en el ILE, SGM. Sin embargo las cirugías clasificadas como "óptimas" por parte del equipo quirúrgico tuvieron mayor ILE (Delta 17 meses) $P=0.038$.

Conclusión: En esta serie de casos presentada, las pacientes con cáncer de ovario en Etapa clínica I tuvieron una mejor supervivencia que las pacientes con Etapa clínica III. Así mismo las cirugías clasificadas como óptimas tuvieron mayor intervalo libre enfermedad y mayor supervivencia libre de enfermedad.

Palabras Claves: NEOPLASIAS OVÁRICAS, SUPERVIVENCIA (SALUD PÚBLICA), OVARIO.

DOI: $10.33821 / 106$

\section{Abstract}

Introduction: Ovarian cancer is a common cause of death among women who develop gynecological neoplasms. Survival depends on clinical factors and the type of surgery. The objective of the present study is to describe the survival of a group of patients diagnosed with ovarian cancer taking into account several factors.

Methods: The present retrospective longitudinal study was conducted in women with ovarian cancer treated at the National Institute of Oncology and Radiobiology, Havana-Cuba between January 1 and December 31, 2005, with follow-up of at least 5 years. The variables were age, histological type of the tumor, clinical stage of the FIGO, degree of cellular differentiation, values of the CA- 125 biomarker, type of surgery performed, response to the chemotherapy used, survival, recurrences. The statistical package used was SPSS 11.0 for Windows. Statistical tests were applied, such as the non-parametric Kruskal-Wallis test, the nonparametric Mann-Whitney test and the Pearson Chi-square test.

Results: There were 29 cases of women with ovarian cancer. Eleven cases (37.9\%) were in children under 45 years of age. In Clinical Stage III, 18 cases (62.1\%). Of undifferentiated degree in 17 cases (58.6\%). 16 cases $(55.2 \%)$ were of the serous histological type. The value of CA-125 was $>35 \mathrm{U} / \mathrm{mL}$ in 13 cases (61.9\%). Women with clinical stage I had a Disease Free Interval (DFI) of 46 months versus 27 months in women with stage III and 12 months in stage IV $(P>0.05)$. The degree of differentiated cellular differentiation statistically determined the best DFI: 55 months versus 27 months in the moderately differentiated degree and 21 months in the undifferentiated degree $(P=0.025)$. The histological type as well as CA-125 levels did not determine statistical differences of DFI, as well as in Average Global Survival (AGS). According to the type of treatment, there were no significant statistical differences in the FID and AGS. However, surgeries classified as "optimal" by the surgical team had a greater DFI (Delta 17 months) $\mathrm{P}=0.038$.

Conclusion: In this case series presented, patients with ovarian cancer in clinical stage I had a better survival than patients with clinical stage III. Likewise, surgeries classified as optimal had greater free disease interval and greater disease-free survival.

Keywords: BREAST NEOPLASMS, NON-HODGKIN LYMPHOMA, SURVIVAL (PUBLIC HEALTH).

DOI: 10.33821/106 


\section{Introducción}

El cáncer de ovario es la causa más común de muerte entre las mujeres que desarrollan neoplasias ginecológicas en países desarrollados. En los Estados Unidos, el riesgo de por vida de padecer cáncer de ovario es aproximadamente $1.4 \%$ ( 1 en 71 ), y el riesgo de morir por esta causa es alrededor de 1 en 95 [1].

En el Instituto Nacional de Oncología y Radiobiología de Cuba (INOR), según un estudio publicado en el año 2009 que se refería al comportamiento del cáncer de ovario en esta Institución en el quinquenio 2001 - 2005, el grupo de edad predominante fue el de 40-59 años y el tipo histológico más frecuente fue el Adenocarcinoma Papilar Seroso dentro de un grupo de 52 pacientes que cumplían los criterios de inclusión en la investigación [2].

El tratamiento del cáncer de ovario se basa en la integración de la cirugía y la quimioterapia. La quimioterapia juega un rol importante en el tratamiento adyuvante y como arma terapéutica en la enfermedad avanzada [3]. La cirugía citorreductiva primaria es la piedra angular en el tratamiento del cáncer de ovario y su mayor factor pronóstico [4].

La cirugía de las enfermedades malignas del ovario se considera la de mayor reto dentro de los procedimientos quirúrgicos en Ginecología. Aunque las tasas de resección óptima en centros con experiencia en Oncología Ginecológica alcanzan hasta un 90 por ciento, la cirugía óptima en instituciones promedio puede lograrse sólo en el 30 al 60 \% en la etapa III/IV del cáncer de ovario [5]. El tratamiento primario para el cáncer de ovario epitelial ha sido tradicionalmente la cirugía, esto se ha basado en el principio de la cirugía citorreductora o cirugía de debulking. En el momento de la intervención quirúrgica, la enfermedad está a menudo diseminada a lo largo de la cavidad peritoneal. Esto hace imposible llevar a cabo la eliminación completa del tumor con un claro margen de tejido normal. Varios estudios han demostrado que el pronóstico en general de las pacientes se mejora si la mayor parte de la enfermedad es eliminada durante la cirugía, comparado con las pacientes a las que no es posible eliminar la enfermedad. La intervención quirúrgica también ha sido utilizada como un procedimiento de intervalo que consiste en realizarla después de un ciclo de quimioterapia. Mientras la cirugía es la modalidad de tratamiento primario para el Cáncer de ovario, la quimioterapia es utilizada en dos escenarios en el manejo de esta enfermedad: primero como tratamiento adyuvante que sigue a la cirugía y segundo como tratamiento neoadyuvante o primario en esas pacientes para las que la cirugía no es aconsejable dada la comorbilidad significativa o por una enfermedad extensamente diseminada.

Los factores pronósticos del cáncer de ovario incluyen la etapa y el grado histológico del cáncer al diagnóstico, la presencia o ausencia de enfermedad residual al concluir la cirugía inicial, el estatus funcional del paciente, la edad, y el uso y no-uso de quimioterapia basada en platino. Cuando el cáncer de ovario es detectado y tratado mientras aún está confinado al ovario (etapa I), la tasa de supervivencia a los 5 años es aproximadamente del $90 \%$, en contraste a la tasa de aproximadamente $33 \%$ cuando la enfermedad es diagnosticada en las etapas III y IV [6].

El cáncer de ovario representa una enfermedad biológica compleja para evaluar. Existen múltiples tipos celulares, grados histológicos y etapas. Hay una gran variación en los 
términos de estadificación quirúrgica y el tamaño y extensión del tumor residual después de la cirugía primaria. Estas variables constituyen un reto que lleva a evaluar con precisión la respuesta a varias modalidades disponibles para el tratamiento que incluyen citorreducción quirúrgica del tumor, quimioterapia y ahora la adición de agentes biológicos. El objetivo del presente estudio es describir la supervivencia de un grupo de pacientes con diagnóstico de cáncer de ovario.

\section{Materiales y Métodos}

Se realizó una investigación descriptiva longitudinal retrospectiva sobre los resultados en la atención a las pacientes con cáncer de ovario, inscritas en el INOR entre el 1 de enero y el 31 de diciembre de 2005. Se analizaron los resultados obtenidos en un período de 5 años. El tipo de muestreo fue no probabilístico de conveniencia, del período estudiado. Se excluyeron casos en los que no se tuvo la información clínica completa.

El grupo de estudio estuvo constituido por la totalidad de las pacientes con confirmación histológica de neoplasia ovárica maligna que se inscribieron en el INOR en el periodo señalado y que fueron seguidas continuamente en este centro entre el 2005 y el 2010. Para obtener la información se realizó una revisión documental de las historias clínicas. Para la recolección de los datos se utilizó un formulario confeccionado para este fin.

Las variables analizadas fueron edad de las pacientes, tipo histológico del tumor, etapa clínica de la FIGO, grado de diferenciación celular, valores del biomarcador CA-125, tipo de cirugía realizada, respuesta a la quimioterapia utilizada, variables relacionadas con la supervivencia, así como con las recurrencias.

Para el análisis estadístico, los datos se almacenaron en un fichero con el programa profesional SPSS (Statistical Package for Social Sciences) versión 11.0 para Windows. Para la descripción de datos se aplicaron medidas como por cientos, media, rango y desviación estándar. Se aplicaron pruebas estadísticas como la prueba no paramétrica de Kruskal-Wallis, la prueba no paramétrica de Mann-Whitney y la prueba de Chi-cuadrado de Pearson. Los niveles de significación estadística fueron con un valor $P<0.05$.

\section{Resultados}

Se analizaron un total de 29 casos de cáncer de ovario confirmado histológicamente. Se excluyeron 3 pacientes por no tener un seguimiento adecuado durante el estudio. Once casos (37.9\%) correspondieron a menores de 45 años de edad. La mayoría de los casos 18 casos $(62.1 \%)$ estuvieron en etapa Clínica III. El grado de diferenciación celular fue indiferenciado en 17 casos (58.6 \%). 16 casos (55.2\%) fueron del tipo histológico seroso. El valor del CA-125 fue $>35 \mathrm{U} / \mathrm{mL}$ en 13 casos (61.9\%) (Tabla 1 ). 
Las cirugías se realizaron con un momento de intervalo en 9 casos (31\%), 17 casos (58.6 $\%$ ) fueron realizados en el propio centro hospitalario (INOR) 7 en resto de los casos se realizó fuera de la institución. El número de casos con recurrencia fue de 9 (31 \%) y 7 casos $(24.1 \%)$ con persistencia de la enfermedad a pesar del tratamiento (Tabla 2).

Sobre la edad la mujeres con edad menor a 45 años tuvieron mejor Intervalo Libre de enfermedad que las mujeres mayores o iguales a 45 años (Delta 20 meses), así mismo presentaron mejor Supervivencia Global media (Delta de 13 meses) y con una leve diferencia en mejor supervivencia libre de enfermedad (Delta 4 \%) (Tabla 1).

Tabla 1. Intervalo libre de enfermedad, supervivencia global media y supervivencia libre de enfermedad a los cinco años según algunos parámetros seleccionados en las pacientes con Cáncer de ovario.

\begin{tabular}{|c|c|c|c|c|}
\hline & No - $\%$ & ILE & $S G M$ & $S L E$ \\
\hline \multicolumn{5}{|l|}{ Edad } \\
\hline$<45$ años & 77 (37.9) & 47.0 & 55.0 & $6(54.5)$ \\
\hline$\geq 45$ años & $78(62.7)$ & 27.0 & 42.0 & $2(17.7)$ \\
\hline \multicolumn{5}{|l|}{ Etapa clínica* } \\
\hline Etapa I & $6(20.7)$ & 46.0 & 62.0 & $5(83.3)$ \\
\hline Etapa // & --- & -- & --- & --- \\
\hline Etapa III & $18(62.1)$ & 27.0 & 46.0 & $3(16.7)$ \\
\hline \multirow[t]{2}{*}{ Etapa IV } & $5(77.2)$ & 12.0 & 34.0 & --- \\
\hline & & $p=0.076$ & $P=0.105$ & $p=0.002$ \\
\hline \multicolumn{5}{|l|}{ Grado de diferenciación celular* } \\
\hline Bien diferenciado & $5(17.2)$ & 55.0 & 60.0 & $5(100)$ \\
\hline Moderadamente diferenciado & $7(24.7)$ & 27.0 & 57.0 & $7(74.3)$ \\
\hline \multirow[t]{2}{*}{ Indiferenciado } & $77(58.6)$ & 21.0 & 40.0 & $2(17.8)$ \\
\hline & & $p=0.025$ & $P=0.109$ & $p=0.000$ \\
\hline \multicolumn{5}{|l|}{ Tipo histológico* } \\
\hline Seroso & $16(55.2)$ & 24.0 & 45.0 & $4(25.0)$ \\
\hline Mucinoso & $3(10.3)$ & 45.0 & 42.0 & $2(66.7)$ \\
\hline Endometrioide & 4 (13.8) & 27.0 & 54.0 & $7(25.0)$ \\
\hline \multirow[t]{2}{*}{ Otro } & $6(20.7)$ & 36.0 & 57.0 & $7(16.7)$ \\
\hline & & $p=0.403$ & $P=0.616$ & $p=0.437$ \\
\hline \multicolumn{5}{|l|}{$C A-125^{* *} \dagger$} \\
\hline$\leq 35 \mathrm{U} / \mathrm{ml}$ & $8(38.7)$ & 35.0 & 57.0 & $4(50.0)$ \\
\hline \multirow[t]{2}{*}{$>35 \mathrm{U} / \mathrm{ml}$} & 13 (61.9) & 23.0 & 42.0 & $2(15.4)$ \\
\hline & & $p=0.243$ & $P=0.210$ & $p=0.222$ \\
\hline
\end{tabular}

ILE: intervalo libre de enfermedad (valor de la media en meses), SGM: supervivencia global media (en meses), SLE: supervivencia libre de enfermedad (porcentaje).

* Prueba no paramétrica de Kruskal-Wallis

** Prueba no paramétrica de Mann-Whitney

†El porcentaje es en base a las pacientes a las que se le realizó la prueba. 
Tabla 2. Características del tratamiento quirúrgico en las pacientes con Cáncer de ovario.

\begin{tabular}{|ll|}
\hline Tipo de tratamiento inicial & valor - no. (\%) \\
\hline Momento de la cirugía & $20(69.0)$ \\
\hline Inicial & $9(31.0)$ \\
De intervalo & \\
Cirugía en otro centro hospitalario & $17(58.6)$ \\
No & $12(41.4)$ \\
Si & $3(25.0) *$ \\
Completa & $9(75.0) *$ \\
Incompleta & $9(31.0)$ \\
Recurrencias o persistencias & $13(44.8)$ \\
\hline Si & $7(24.1)$ \\
\hline No & \\
\hline Persistencia & \\
\hline
\end{tabular}

HTA: Histerectomía total abdominal, SOB: Salpingo-oforectomía bilateral, 0: Omentectomía, AUL: Anexectomía unilateral.

* El porcentaje es de acuerdo a la cantidad de pacientes que recibieron tratamiento quirúrgico en otro centro hospitalario.

Tabla 3: Intervalo libre de enfermedad, supervivencia global media y supervivencia libre de enfermedad a los cinco años según el tipo de tratamiento quirúrgico recibido en las pacientes con Cáncer de ovario.

\begin{tabular}{|c|c|c|c|c|}
\hline & no- $\%$ & ILE & $S G M$ & $S L E$ \\
\hline \multicolumn{5}{|c|}{ Tipo de tratamiento* } \\
\hline $\mathrm{HTA}+\mathrm{SOB}+\mathrm{O}$ & $13(44.8)$ & 38.0 & 52.0 & $6(75.0)$ \\
\hline $\mathrm{HTA}+\mathrm{SOB}$ & $7(24.1)$ & 25.0 & 45.0 & $2(25.0)$ \\
\hline AUL & --- & --- & --- & --- \\
\hline \multirow[t]{2}{*}{ Irresecable } & $9(31.0)$ & 19.0 & 42.0 & --- \\
\hline & & $P=0.107$ & $P=0.410$ & $P=0.059$ \\
\hline \multicolumn{5}{|c|}{ Clasificación de la cirugía** } \\
\hline Óptima & $13(44.8)$ & 38.0 & 52.0 & $6(75.0)$ \\
\hline \multirow[t]{2}{*}{ No óptima } & $16(55.2)$ & 21.0 & 43.0 & $2(25.0)$ \\
\hline & & $P=0.038$ & $P=0.182$ & $P=0.044$ \\
\hline \multicolumn{5}{|c|}{ Cirugía en otro centro**† } \\
\hline Completa & $3(25.0)$ & 47.0 & 59.0 & $2(50.0)$ \\
\hline \multirow[t]{2}{*}{ Incompleta } & $9(75.0)$ & 26.0 & 48.0 & $2(50.0)$ \\
\hline & & $P=0.562$ & $P=0.926$ & $P=0.278$ \\
\hline
\end{tabular}

HTA: Histerectomía total abdominal, SOB: Salpingooforectomía bilateral, 0: Omentectomía, AUL: Anexectomía unilateral. ILE: intervalo libre de enfermedad (valor de la media en meses)

SGM: supervivencia global media (en meses), SLE: supervivencia libre de enfermedad (por ciento).

* Prueba no paramétrica de Kruskal-Wallis, ** Prueba no paramétrica de Mann-Whitney

†El porcentaje es de acuerdo a la cantidad de pacientes que recibieron tratamiento quirúrgico en otro centro hospitalario.

De acuerdo a la etapa clínica las mujeres con etapa clínica más temprana I, demostraron mejor Intervalo Libre de la enfermedad (46 meses) con respecto a las mujeres en etapa III 
(27 meses) y etapa IV (12 meses) estadísticamente no significativo debido al número de casos reducidos en la etapa IV (Tabla 1). El grado de diferenciación celular Bien diferenciado determinó estadísticamente el mejor intervalo libre de enfermedad 55 meses versus 27 meses en el grado moderadamente diferenciado y de 21 meses en el grado indiferenciado $(P=0.025)$ (Tabla 1$)$.

El tipo histológico así como los niveles de CA-125 no determinaron diferencias estadísticas de Intervalo libre de enfermedad, así como en Supervivencia Global media (Tabla 1).

Según el tipo de tratamiento no existió diferencias estadísticas significativas en el Intervalo Libre de enfermedad, Supervivencia global media. Sin embargo las cirugías clasificadas como "óptimas" por parte del equipo quirúrgico tuvieron mayor intervalo libre de Enfermedad (Delta 17 meses) $P=0.038$ (Tabla 3).

Tabla 4. Características de las recurrencias durante un período de 5 años (2005-2010) en las pacientes con cáncer de ovario.

\begin{tabular}{|c|c|c|c|c|c|c|}
\hline Edad & Histología & $\begin{array}{l}\text { Grado de } \\
\text { diferenciación }\end{array}$ & Ascitis & QTP & Sitio & Conducta llevada a cabo \\
\hline 61 & ED & Moderado & No & Si & $\begin{array}{l}\text { Ganglios } \\
\text { para- } \\
\text { aórtico }\end{array}$ & $\begin{array}{l}\text { 1ra y 2da recurrencia } \\
\text { Carboplatino /Paclitaxel. } \\
\text { Actualmente con HR3* }\end{array}$ \\
\hline 49 & Seroso & Pobre & Si & Si & $\begin{array}{l}\text { Hígado / } \\
\text { pulmón }\end{array}$ & $\begin{array}{l}\text { Carboplatino } \\
\text { Ciclofosfamida } \\
\text { Pleurodesis con talco }\end{array}$ \\
\hline 60 & Seroso & Pobre & Si & Si & $\begin{array}{l}\text { Hígado / } \\
\text { peritoneo } \\
\text { pélvico }\end{array}$ & $\begin{array}{l}\text { Carboplatino } \\
\text { Ciclofosfamida }\end{array}$ \\
\hline 45 & Seroso & Moderado & Si & Si & $\begin{array}{l}\text { Peritoneo } \\
\text { pélvico }\end{array}$ & $\begin{array}{l}\text { Carboplatino / Paclitaxel } \\
\text { Colostomía transversa }\end{array}$ \\
\hline 68 & ED & Moderado & Si & Si & $\begin{array}{l}\text { Peritoneo } \\
\text { pélvico }\end{array}$ & $\begin{array}{l}\text { Carboplatino / } \\
\text { Paclitaxel }\end{array}$ \\
\hline 39 & ID & Pobre & Si & -- & $\begin{array}{l}\text { Peritoneo } \\
\text { pélvico }\end{array}$ & $\begin{array}{l}\text { Datos no } \\
\text { disponibles }\end{array}$ \\
\hline 38 & Seroso & Pobre & Si & Si & $\begin{array}{l}\text { Peritoneo } \\
\text { pélvico } \\
\text { Metástasis } \\
\text { cerebral }\end{array}$ & $\begin{array}{l}\text { Carboplatino/Adriamicina/ } \\
\text { Ciclofosfamida }\end{array}$ \\
\hline 55 & Seroso & Pobre & Si & Si & $\begin{array}{l}\text { Peritoneo } \\
\text { pélvico } \\
\text { Metástasis } \\
\text { a piel }\end{array}$ & $\begin{array}{lll}\text { Cirugía en } & \text { varias } \\
\text { oportunidades y } & \text { varios } \\
\text { esquemas de QTP } & \end{array}$ \\
\hline 42 & Seroso & Pobre & Si & Si & $\begin{array}{l}\text { Peritoneo } \\
\text { pélvico } \\
\text { Oclusión } \\
\text { intestinal }\end{array}$ & $\begin{array}{l}\text { Cirugía } \\
\text { Carboplatino / Paclitaxel }\end{array}$ \\
\hline
\end{tabular}

ED: Endometroide. ID: Indiferenciado, QTP: Quimioterapia. *HR3: Anticuerpo monoclonal contra el receptor del factor de crecimiento epidérmico. 


\section{Discusión}

Se analizaron un total de 31 historias cínicas con diagnóstico de cáncer de ovario confirmado histológicamente; de ellas se excluyeron 3 pacientes por no tener un seguimiento adecuado durante el estudio. Como se puede ver en la tabla 1 las pacientes con una edad inferior a los 45 años tuvieron un intervalo libre de enfermedad (ILE), una supervivencia global media (SGM) y una supervivencia libre de enfermedad (SLE) superior que las que sobrepasaban esta edad. Las pacientes que se encontraban en etapa I de la enfermedad tuvieron un ILE medio de 46 meses, con una SGM de 62 meses y una SLE del $83.3 \%$, sin embargo aquellas que se encontraban en etapa IV el ILE fue sólo de 12 meses, con una SGM de 34 meses y ninguna de las 5 pacientes en esta etapa sobrevivieron libre de enfermedad. Se observaron diferencias altamente significativas en la relación entre la etapa de la enfermedad en la que se encontraban las pacientes y la SLE y muy cercanas a los límites de significación en lo referente al ILE; no se tienen evidencias de que existieran diferencias en cuanto a las etapas respecto a la SGM. Los tumores bien diferenciados presentaron un ILE muy por encima que los tumores moderadamente diferenciados y que los indiferenciados, también fue así en lo referente a SGM.

Todas las pacientes con tumores bien diferenciados habían sobrevivido libres de enfermedad al concluir el estudio. Se encontraron diferencias altamente significativas entre el grado de diferenciación celular y la SGM y cercanas a los límites de significación para el ILE, no fue así para la SGM. Los mayores ILE se presentaron en las pacientes con tumores mucinosos con una SLE $66.7 \%$, sin embargo la SGM fue algo superior en la histología endometrioide. En ninguna de las histologías se encontró significación estadística. El valor normal del biomarcador $C A-125$ se relacionó con un ILE y una SGM superior, tanto es así que la mitad de estas pacientes sobrevivieron libres de enfermedad. Sin embargo para el ILE, la SGM y el SLE no se tienen evidencias de que difieran estadísticamente según la prueba no paramétrica de Mann-Whitney. En uno de los estudios consultados se encontró una significación estadística entre SLE y una edad superior a la media, que para este trabajo era de 60 años, también los tumores indiferenciados fueron más frecuentes aunque ellos no encontraron significación estadística respecto a supervivencia [7].

Según la literatura médica actual, de las mujeres en etapa I de la enfermedad la supervivencia aproximada a los 5 años es del 95\%, mientras para aquellas que se diagnostican en etapa III o IV la supervivencia cae hasta un 20 a 30\% [8]. En otra de las investigaciones revisadas se encontró significación estadística entre el grado de diferenciación celular del tumor y la SLE, en esta los tipos histológicos endometrioide y un valor normal del marcador $C A-125$ se asociaron a porcentajes mayores de SLE. Los elementos relacionados con la morfología e histología del tumor, que incluye la arquitectura y el grado de la lesión son importantes variables de pronóstico, los tipos histológicos no se han relacionado generalmente con la significación en el pronóstico aunque estudios recientes han sugerido que los carcinomas de células claras si se asocian con un peor pronóstico que los otros tipos histológicos $[9,10]$.

En la tabla 2 se analiza todo lo referente a la relación entre el tratamiento quirúrgico llevado a cabo y los resultados obtenidos en cuanto a ILE, SGM y SLE. Las pacientes que no fue 
posible realizarle ningún proceder, excepto la toma de muestra para estudio histológico, tuvieron un ILE medio de sólo 19 meses; muy bajo respecto a las que recibieron una cirugía completa. También la SGM estuvo por encima en las pacientes que se les hizo el procedimiento quirúrgico estándar y estas tuvieron una SLE del $75 \%$. No obstante cuando se aplicaron pruebas estadísticas como la no paramétrica de Kruskal-Wallis sólo se encontró una diferencia cercana al límite de significación en el caso de la SLE. Cuando se analizó la clasificación de la cirugía se encontraron mejores resultados en las pacientes que pudieron recibir una cirugía óptima las que alcanzaron un ILE medio de 38 meses, con una SGM de 52 meses y el $75 \%$ sobrevivieron libre de enfermedad. Aquí se encontró asociación estadísticamente significativa en lo que respecta a ILE y SLE.

De las pacientes que fueron intervenidas quirúrgicamente en otro centro hospitalario solamente se encontraron diferencias respecto al ILE que fue de 47 meses en las que la cirugía fue completa en relación a las que la cirugía fue incompleta que permanecieron libres de enfermedad sólo una media de 26 meses. No hubo diferencias en cuanto a SGM y SLE. No se encontró significación estadística en relación a la cirugía en otro centro y los resultados en cuanto a ILE, SGM ni SLE. Históricamente se ha sugerido que existe una relación inversa entre el diámetro tumoral residual y la supervivencia, y últimamente se ha llegado a la conclusión de que la cirugía provee un beneficio óptimo cuando es extirpado todo tumor macroscópico; esta observación inicial se ha sido confirmada por múltiples estudios subsecuentes y se ha convertido en el paradigma actual de tratamiento [7]. Chi et a/ en una publicación reciente analizaron la influencia de la cirugía del abdomen superior respecto al ILE y supervivencia global en pacientes con cáncer de ovario, tubárico o peritoneal en etapa IIIC y IV y encontraron una tasa de citorreducción significativamente más alta en las pacientes que se les llevó a cabo una cirugía extendida al abdomen superior [11]. En Europa la cirugía óptima sólo se logra en una minoría de las pacientes en etapas avanzadas causado por la falta de un entrenamiento formal en las técnicas quirúrgicas utilizadas frecuentemente en la citorreducción, de hecho la proporción de pacientes operadas por un Ginecólogo oncólogo con entrenamiento en cirugía extensa varía entre el $0 \%$ y el $95 \%$, los datos de la literatura europea el porcentaje de enfermedad residual $\leq 1 \mathrm{~cm}$ durante la cirugía primaria oscila entre el $37.8 \%$ y el $81 \%$ [12].

La relación existente entre la respuesta de estas pacientes a la QTP de acuerdo a si se pudo o no lograr una cirugía óptima se muestra en la tabla 3. En aquellas enfermas que la respuesta fue parcial o no respondieron a la medicación, el 73.3 \% la cirugía no había sido óptima. Sin embargo de las que si respondieron a los esquemas planificados el $58.3 \%$ se les había podido practicar una citorreducción óptima. Sin embargo no hubo una relación significativa desde el punto de vista estadístico entre las mencionadas variables (Chicuadrado de Pearson: $5.347 \mathrm{p}=0.069)$ Numerosos estudios, que incluyen aquellos realizados por el Gynecoloy Oncology Group (GOG) han mostrado tasas superiores de respuesta a la QTP, un mayor intervalo libre de progresión y una supervivencia media superior en pacientes que se logró llevar a cabo una citorreducción óptima [8]. Estas observaciones se apoyan en estudios básicos que sugieren que las grandes masas tumorales tienen áreas anóxicas pobremente perfundidas que no son accesibles a los agentes citotóxicos, además los tumores de mayor tamaño pueden tener una proporción superior de células en etapa de reposo; estas células no proliferativas pueden ser menos sensibles a los agentes citotóxicos [13]. 
Como se puede apreciar en la tabla 4, nueve pacientes sufrieron una o más recidivas tumorales durante el periodo de cinco años en el que se enmarcó el estudio. Esto representó el $31.0 \%$ del total. El tipo histológico que se relacionó más frecuentemente con estas recaídas fueron los tumores epiteliales, fundamentalmente los subtipos serosos con seis pacientes. Los tumores pobremente diferenciados se relacionaron con mayor frecuencia con las recaídas y fueron clasificados así en seis de las nueve pacientes, en ninguno de estos episodios los tumores eran bien diferenciados. La ascitis fue un hallazgo muy frecuente y se presentó en casi la mayoría de los casos. En dos de estas pacientes se presentaron más de un episodio de recaída. El sitio más frecuentemente afectado fue el peritoneo pélvico que se vio comprometido en siete de éstas. Todos, excepto uno de los casos en el que no se encontraron los datos adecuados que permitieran su análisis, recibieron QTP como tratamiento único o combinado con la cirugía para la eliminación de la lesión recidivante. La enfermedad recidivante se clasifica en temprana o tardía. Menos del $10 \%$ de las pacientes consideradas platino-resistentes que recurren después de una remisión completa anterior tendrán igual resultado una vez más, y su supervivencia media es menor de un año. Las consideradas como platino-sensible pueden tener una tasa de respuesta del 20 al $60 \%$ y la probabilidad de responder se asocia a un ILE más prolongado entre la terminación de la terapia primaria y la enfermedad recurrente [8]. Diferentes estudios retrospectivos han mostrado una mejora en la supervivencia de pacientes que se les ha practicado una cirugía citorreductora secundaria, las candidatas óptimas para tal proceder parecen ser aquellas que son sensibles al platino [13].

\section{Conclusiones}

En esta serie de casos presentada, las pacientes con cáncer de ovario en Etapa clínica I tuvieron una mejor supervivencia que las pacientes con Etapa clínica III. Así mismo las cirugías clasificadas como óptimas tuvieron mayor intervalo libre enfermedad y mayor supervivencia libre de enfermedad.

\section{Agradecimientos}

Reconocemos a las personas que participaron indirectamente en el estudio tales como el personal técnico, personal del Instituto Nacional de Oncología y Radiobiología, La HabanaCuba.

\section{Información adicional}

\section{Abreviaturas}

AUL: Anexectomía unilateral.

HTA: Histerectomía total abdominal.

ILE: intervalo libre de enfermedad (valor de la media en meses). 
0: Omentectomía.

SGM: supervivencia global media (en meses).

SLE: supervivencia libre de enfermedad (porcentaje).

SOB: Salpingooforectomía bilateral.

Nota del Editor

La Revista Oncología Ecu permanece neutral con respecto a los reclamos jurisdiccionales en mapas publicados y afiliaciones institucionales.
Archivos Adicionales

Ninguno declarado por los autores.

\section{Fondos}

Los fondos de la investigación fueron propios de los autores del presente artículo.

\section{Disponibilidad de datos y materiales}

Existe la disponibilidad de datos bajo solicitud al autor de correspondencia. No se reportan otros materiales.

\section{Contribuciones de los autores}

IGA, ALL, RPM realizaron la idea de investigación, revisión bibliográfica. MSP, REN, KAVDO recolección de datos, escritura del artículo, análisis crítico del artículo. IGA realizó las correcciones editoriales. Todos los autores leyeron y aprobaron la versión final del artículo.

Aprobación de ética y consentimiento para participar

No aplica a este estudio.

\section{Consentimiento para publicación}

No aplica.

\section{Información de los autores}

Iván García Alonso. Médico del Hospital Universitario "Celestino Hernández Robau". Santa

Clara- Cuba. Email: igarcia@hchr.vcl.sld.cu Teléfono (53) 042210023. https://orcid.org/0000-0002-3229-1178 
Alejandro Linchenat Lambert. Médico del Instituto Nacional de Oncología y Radiobiología (INOR). La Habana-Cuba.

Roberto Pérez Martínez. Médico del Hospital "Gustavo Aldereguia Lima" Cienfuegos-Cuba.

Mario Silveira Pablos. Médico del Instituto Nacional de Oncología y Radiobiología (INOR). La Habana-Cuba.

Roberto Esperón Noa. Médico del Instituto Nacional de Oncología y Radiobiología (INOR). La Habana-Cuba.

Kenia Aguilar Vela de Oro. Instituto Nacional de Oncología y Radiobiología (INOR). La Habana. Cuba.

Revisiones por pares

Acceda a la revisión de pares académicos en el siguiente enlace: https://publons.com/review/4078313

\section{Referencias}

Abreviaturas en la referencias

DOI: Digital Object

Identifier

PMID: PubMed Identifier

SU: Short URL
1. Jemal A, Siegel R, Ward E, Hao Y, Xu J, Thun MJ. Cancer statistics 2009. CA Cancer J Clin 2009;59: 22549.

2. Rodríguez Reigosa JE. Cáncer de ovario en el Instituto Nacional de Oncología y Radiobiología de Cuba: 2001 a 2005. Rev Cubana Cir [online]. 2009, vol.48, n.1, pp. 0-0. ISSN 0034-7493.

3. Colombo N, Peiretti M, Parma G, Lapresa M, Mancari R, Carinelli S, Sessa C, Castiglione M. Newly diagnosed and relapsed epithelial ovarian carcinoma: ESMO Clinical practice guidelines for diagnosis, treatment and follow-up on Behalf of the ESMO Guidelines Working Group, 2010.

4. Bristow RE, Tomacruz RS, Armstrong DK, Trimble EL, Montz FJ. Survival effect of maximal cytoreductive surgery for advanced ovarian carcinoma during the platinum era: a meta-analysis. $\mathrm{J}$ Clin Oncol 2002;20:1248-59.

5. Covens AL. A critique of surgical cytoreduction in advanced ovarian cancer. Gynecol Oncol 2000;78:26974.

6. Clarke-Pearson DL. Screening for ovarian cancer. N Engl J Med 2009 36;2:170-7.

7. Peiretti M, Zanagnolo V, D. Aletti G, Bocciolone L, Colombo N, Landoni F et al. Role of maximal primary cytoreductive surgery in patients with advanced epithelial ovarian and tubal cancer: Surgical and oncological outcomes. Single institution experience. Gynecol Oncol 2010; 119:259-64.

8. Gehring PA, Alvarez Secord A. Gynecologic Oncology. 1ra ed. Texas; 2009

9. Naglea CM, Olsena CM, Webba PM, Jordana SJ, Whitemana DC, Ade le C. Greena A le C et al. Endometrioid and clear cell ovarian cancers - A comparative analysis of risk factors. Eur $\mathrm{J}$ Cancer 2008;44:2477-84.

10. Berek JS, Natarajan S. Ovarian and Fallopian Tube Cancer. En Berek JS. Berek \& Novak's Gynecology, 14th Ed 2007 Lippincott Williams \& Wilkins. p 1457-548. 
11. Chi DS, Eisenhauer EL, Zivanovic 0 , et al. Improved progression-free and overall survival in advanced ovarian cancer as a result of a change in surgical paradigm. Gynecol Oncol 2009;114(1):26-31.

12. Verleye L, Vergote I, van der Zee AG. Patterns of care in surgery for ovarian cancer in Europe. Eur J Surg Oncol 2010;36(Suppl 1):108-14.

13. van Nagel JR, Gershenson. Ovarian cancer: etiology, screening, and surgery. En Rock JA, Jones HW. Te Linde's Operative Gynecology. 10ma ed Lippincot Williams \& Wilkins; 2008. p 1307-39. 